\title{
3D RECONSTRUCTION AND IMAGE PROCESSING OF ANTHROPOLOGICAL ARCHAEOLOGICAL FINDINGS
}

\author{
A.V. Gaboutchian ${ }^{1 *}$,V. A. Knyaz ${ }^{2,3}$, N.A. Leybova ${ }^{4}$, H.Y. Simonyan $^{5}$, M.M. Novikov ${ }^{6}$, S.V. Apresyan ${ }^{1}$, S.A. Cherebylo ${ }^{6}$, G.R. Petrosyan ${ }^{7,8}$ \\ ${ }^{1}$ Peoples Friendship University of Russia, 117198, Moscow, Russia armengaboutchian@ mail.ru; dr.apresyan@gmail.com \\ 2 State Research Institute of Aviation System (GosNIIAS), 125319 Moscow, Russia - knyaz@ gosniias.ru \\ ${ }^{3}$ Moscow Institute of Physics and Technology (MIPT), Dolgoprudny, Russia \\ ${ }^{4}$ Institute of Ethnology and Anthropology RAS, Moscow, Russia - nsuvorova@mail.ru \\ ${ }^{5}$ Scientific Research Centre of the Historical and Cultural Heritage, Yerevan, Armenia - haksimon@gmail.com \\ ${ }^{6}$ Research Center Crystallography and Photonics RAS, Shatura, Russia - (novikov,svetlana.cherebylo)@ rambler.ru \\ ${ }^{7}$ Plekhanov Russian University of Economics Yerevan branch, Yerevan, Armenia \\ ${ }^{8}$ International Scientific-Educational Centre of NAS RA, Yerevan, Armenia - petrosyan_gohar@list.ru
}

\section{Commission II, WG II/10}

KEY WORDS: 3D reconstruction, 3D surface analysis, 3D prototyping, odontology

\begin{abstract}
:
A wide variety of methods are used in archaeological research today, including 3D imaging techniques (photogrammetry) which are involved at different stages starting from explorations preceding excavation to multiple studies. The archaeologically obtained material includes anthropological findings, among which odontological (related to human teeth) are of interest as they are composed of resistible tissues (hence are preserved well) and can serve for biological as well as historical interpretations. However, among the methods employed in odontological studies some are destructive and bring to unwelcome irreversible changes or even complete loss of the analysed samples. However, the existing and rapidly-developing techniques, especially, referring to 3D imaging and prototyping, suggest different approaches which can facilitate avoiding undesirable consequences of invasive methods of research. Thus they can provide for either preservation of findings through development and application of non-invasive study techniques, or, at least, preserve data referring the findings which have to be destroyed in order to receive valuable, in terms of research, information. It is shown on the example of the studied mandibular fragment from the Early Bronze archaeological site of Shengavit how multidisciplinary cooperation and the described workflow contribute to preservation of information regarding the finding and possible restoration of its original features. An effective communication between different professionals was provided due to implementing non-contact measurements techniques, obtaining and processing $3 \mathrm{D}$ images and $3 \mathrm{D}$ printing.
\end{abstract}

\section{INTRODUCTION}

Non-contact measurement techniques are widely implemented today on various stages of archaeological research (starting from the initial phases of excavations to documenting, analysing and restoration of findings) and preserving cultural heritage (Badiu et al., 2015; Chibunichev et al., 2018; Scianna and La Guardia, 2018; Vasilyev et al, 2019). Usually, the material used for studies in archaeology, anthropology or palaeoanthropology is unique and requires significant effort to obtain, preserve as well as to study. And here techniques taken from different academic and practical disciplines can reveal more useful applications including described in this paper restorative workflow.

\section{HISTORICAL BACKGROUND}

The currently described case relates to anthropological material from Shengavit an ancient settlement, spread over more than 6 hectares of land, located in City of Yerevan, the capital of Armenia. Starting with short historical background, we should mention that excavations at this archaeological site have been carried during the last century by E. Bayburtyan and decades later by S. Sardaryan. From 2000 until now, expeditions are led by Dr Hakob Simonyan (Scientific Research Centre for Historical and

\footnotetext{
* Corresponding author
}

Cultural Heritage of the Ministry of Culture of the Republic of Armenia); twice joint Armenian-American expeditions have been held (co-headed by H. Simonyan and M. Rothman). The excavations attest that the settlement was founded in the 4th millennium $\mathrm{BC}$ and remained inhabited until 25 th century BC. The excavations have revealed a remarkable picture of urban development, namely a densely built-up area comprising a number of buildings with round and rectangular layouts, streets, a temple complex, a defense system consisting of walls, bastions, towers, a secret passage, household pits and two-storey silos with a total storage capacity of at least 40 tonnes of grain (Figure 1).

According to reconstructions, Shengavit had a population of about 5,000 . Its sizable population, sophisticated urban design featuring monumental architecture (walls fortified with towers, residential dwellings, buildings of cult and ample grain stores), symbols of power, such as staff pommels, specimens of fine ornaments and adornments made of gold and semi-precious gemstones, the existence of a single unit of measurement for weight, advanced craftsmanship, irrigated agriculture, the fact of being surrounded by satellite settlements and other circumstances suggest that Shengavit bears a number of features consistent with the Ancient Near Eastern Town concept (Simonyan, 2015; Simonyan, 2018). Outside the citadel walls, a tomb field (necropolis) was formed in an abandoned suburb of the settlement around 27-25 centuries BC, where tombs of people belonging to different social classes and 


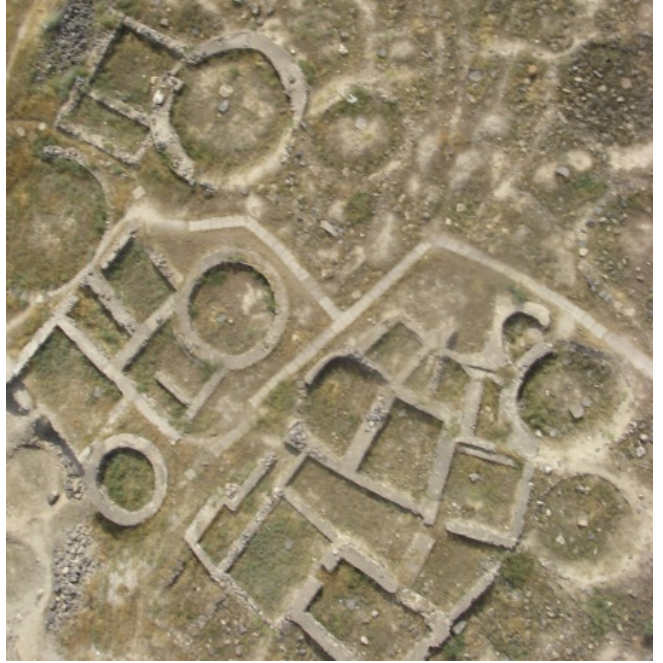

Figure 1. Shengavit; aerial view

complex burial rituals have been documented. The dead were interred in a dismembered state. Thus findings from necropolis are of particular interest in our study: it is a divided in half, according to a funeral rite, mandible with complete set of teeth of dental semi-arch, which has been discovered in 2005 in the 4th tomb (Figure 2).

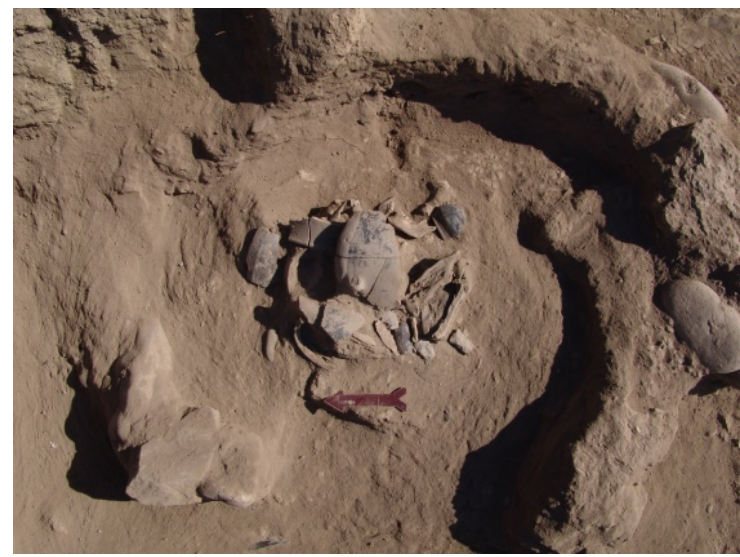

Figure 2. Shengavit: excavations of the 4 th tomb

\section{ODONTOLOGICAL CONSIDERATIONS}

It should be mentioned that highly-mineralised dense dental tissues possess resistibility to damaging factors, which often ensures preservation of teeth among archaeological findings. Thus anthropological study, and especially its odontological part, cannot be divided from archaeology; teeth can be analysed morphologically for taxonomic, phylogenetic or other assessments (Le Luyer et al., 2017; Mahajan 2019; Martin et al., 2017); they serve also for radiocarbon dating, genetic, isotope or other analyses (Foreshaw, 2014; Irish and Scott, 2015). The studied anthropological material was represented by mandible right fragment (involving left part of mandibular symphysis and with partially preserved coronoid and condylar processes) with complete set of right half of dental arch and isolated upper right medial incisor. Despite the fact that the most informative part in anthropological research is the skull, we should mention that mandibular fragment still can possess a potential to provide essential information in anthropological, archaeological of other kind of research or reconstruction (Benazzi et al., 2011). Nevertheless, there are studies providing essential information but require implementing of invasive approaches, and two teeth from the findings were sent to laboratories to conduct genetic and radiocarbon dating analyses, which, in fact, brought to their loss. This refers to separately found upper right medial incisor (1.1 (I1d)) and lower lateral incisor (4.2 (I2d)) from the mandible fragment (Figure 3).

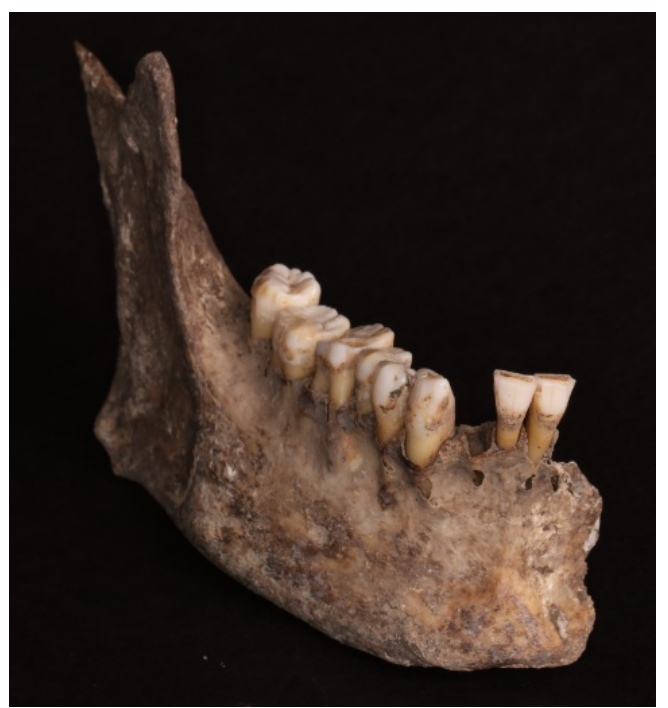

Figure 3. Shengavit: excavations of the 4 th tomb

\section{3D IMAGING}

Despite the fact that the odontological part of studies, including traditional visual odontological description of teeth, as well as manual and automated digital measurements (odontometry), of the finding had been launched prior to starting the genetic analysis and radiocarbon dating and sending teeth to distant laboratories, they were not fully completed by that time. Nevertheless, we already possessed a set of the samples 3D images obtained through different optical and x-ray scanning techniques: conebeam computed tomography (CBCT), intraoral dental scanning and $\mathrm{x}$-ray microtomography of a separate tooth (lower right first premolar). However the most informative image regarding the initial condition of the complete finding (not its parts) was pilot CBCT scan with voxel size of $0.2 \times 0.2 \times 0.2(\mathrm{~mm})$ and FoV (height $\mathrm{x}$ diameter) of $100 \times 85(\mathrm{~mm})$ (Figure 4), though detailed odontological studies normally require relatively higher resolution images. The mentioned higher resolutions are required not only in terms of more precise measurements but for obtaining of exact copies of unique findings, as development of diagnostic methods in medical/dental imaging and anthropology (Sam et al., 2019; Uldin, 2017). In addition improvements in 3D printing techniques provide for more detailed studies in palaeoanthropology and dental anthropology, dentistry and other applications (Ahangar et al., 2019; Brown et al., 2018; Fiorenza et al., 2018). However the presented workflow describes the process of restoration of missing tooth (lower right lateral incisor) on the Early Bronze Age palaeoanthropological sample from Shengavit on the basis of information that we possesed.

\section{METHOD}

Odontological part of the current sample study includes two approaches. The first is based on visual description and manu- 


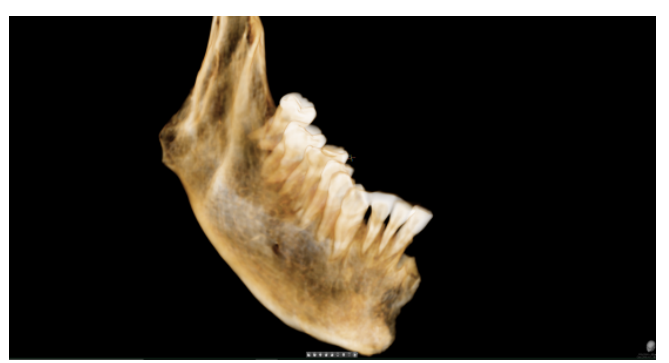

Figure 4. Shengavit: excavations of the 4 th tomb

ally conducted measurements. The used methods were established in works of prominent Soviet/Russian anthropologists and odontologists Mikhail Gerasimov and Alexandr Zubov (Zubov, 1968, 2006; Gerasimov, 1955) and can be integrated with widely accepted and traditional approaches to anthropological research also to this day. Referring to measurements of teeth by calipers, they allow obtaining maximal parameters in two directions: mesiodistal and vestibular-lingual. However, progress in 3D imaging and digital image processing provides for development of techniques on a new level (Knyaz et al., 2008, Knyaz, 2012). Thus automated digital odontometry is the other approach to measurements of teeth in the current study, or even not teeth but rather their 3D images. Fully automated algorithms based on morphological interpretations allow orientation, sectioning and measurements of a wide variety of different linear, angular, area parameters as well as calculation of coefficients and parameter ratios (Gaboutchian et al., 2017, Gaboutchian et al., 2019). Landmark setting on 3D surfaces and 2D contours does not require much effort or profound skills in odontometry or anthropology from the operator.

For launching the described restoration procedures the anthropological sample was scanned on CBCT imaging device PaX-i 3D (Vatech, Korea), designed for dental diagnostic procedures. Due to the sample size (diameter $\mathrm{x}$ height $121 \times 69, \mathrm{~mm}$ ) and limitations of field of view settings for high resolution CBCT images, a series of scans were conducted with voxel size: $0.08 \times 0.08 \times 0.08$ $(\mathrm{mm})$ and FoV (height $\mathrm{x}$ diameter) 50 x $50(\mathrm{~mm})$ with manually adjusted peak kilovoltage and tube current (Figure 5).

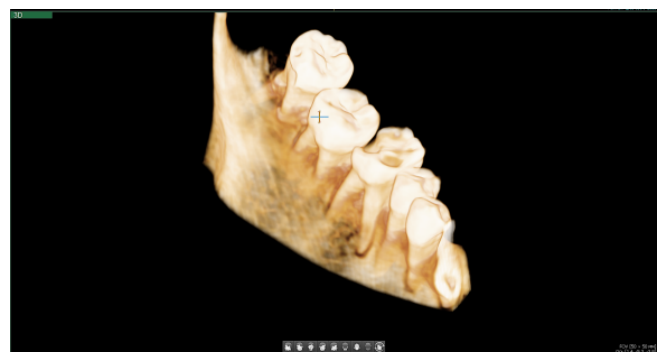

Figure 5. Higher resolution CBCT scan of mandible fragment part

The highest possible resolution for the complete mandible fragment imaging that could be achieved on the devise was $0.2 \times 0.2 \mathrm{x}$ $0.2(\mathrm{~mm})$ voxel size at FoV (height $\mathrm{x}$ diameter) $100 \times 850(\mathrm{~mm})$. All scans were obtained in a single position of the sample to minimize possible distortions and avoid displacement of teeth in their sockets.

The studied sample tomographic data was processed in .dcm file format in Mimics software (Materialise, Belgium). Image processing included artefact removal and optimal grey level adjust- ment (Figure 6) and subsequent generation of precisely reconstructed 3D models of the studied object in .stl file format.

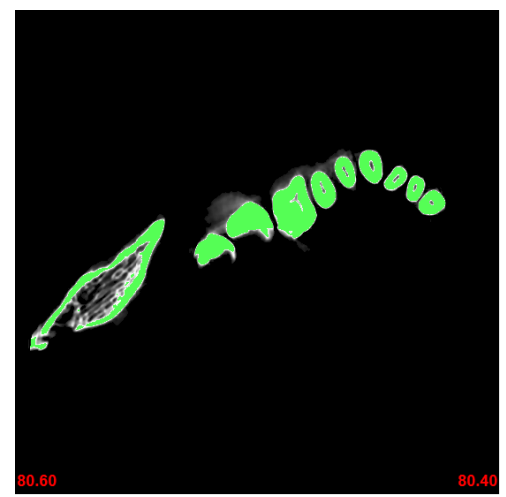

Figure 6. Tomographic slice with specified threshold level for digital model generation

Correct detection of object boundaries was conducted in accordance with details of the tomographic imaging process; after finishing the delimitation process the model reconstruction precision corresponded to the degree of initial scan resolution.

On the next stage, after generating 3D models, they were used for virtual reconstructions of the studied object by means of Magics software (Materialise, Belgium). At the same time, taking into consideration the principal aim of the current study, segmentation of the incisor, that had been sent to a distant laboratory for DNA analysis, was conducted (Figure 7).
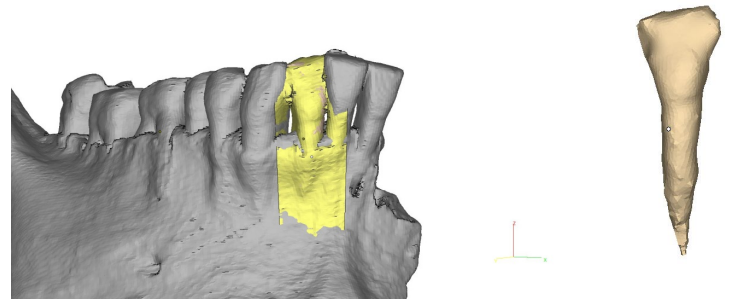

Figure 7. Segmentation of the incisor fragment from the 3D image (left) and the segmented incisor (right)

Thereafter higher resolution scans were combined together for obtaining more detailed mandible fragment image generation. This provided for reconstruction of the complete studied object on a higher resolution level (Figure 8). These models were then used for virtual reconstruction and 3D printing on SLA device LS150 designed at the Institute on Laser and Information Technologies of Russian Academy of Sciences (Vnuk et al., 2019) and NextDent 5100 (3D Systems) printer with maximal resolution of 1920 x 1080 pixels and pixel pitch 65 m (390.8 effective PPI). This provided for realistic reconstruction of lost (for research reasons) odontological material.
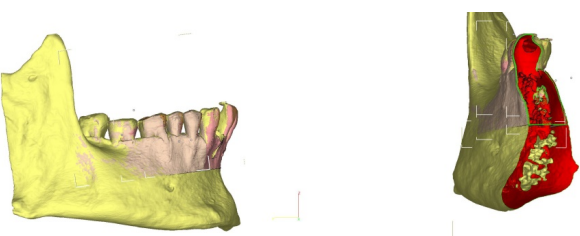

Figure 8. Combined higher resolution CBCT scans: general view (a) and transverse section (b) 


\section{RESULTS}

\subsection{Odontometry}

Without expanding on complete odontological visual study of the sample, we present description of the most massive, distally positioned tooth with moderate degrees of attrition and morphological variability second molar; on the other hand it is of interest in terms of methodological approaches to digital measurements (Figure 9). Thus the second molar has a tendency to reduction: it has four cusps and Y-shaped groove pattern (the third molar is Y5 type). The second and third molars do not possess ridges on their trigonids: neither distal, nor epicristid and mesial ridges. No enamel extension is detected on the first and third molars, while it is found on the second (level 5). Worthy of note is significant disharmony in levels of attrition of the first molar if compared to other teeth: the first molar is the leader in attrition rates. However, we do not possess enough data to judge clearly the reasons of the status observed, besides the fact that these teeth are first to erupt in human permanent dentition normally at age of 6 . According to the attrition degree the mandible belonged to an adult individual (adultus II), presumably male.

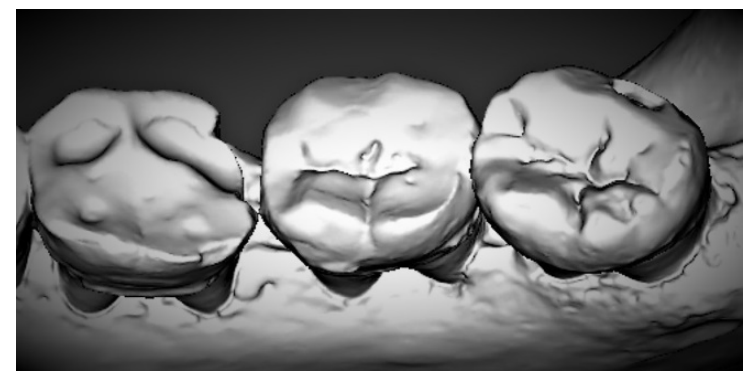

Figure 9. Group of molars; the second molar is in the middle position

The teeth from the Shengavit burial mandibular fragment have small absolute dimensions. Thus, according to mesio-distal crown diameter (MD in Table 1) the second molar can be referred to the category of small teeth (Zubov, 1968), the third molar to medium. Vestibular-lingual diameters (VL in Table 1) of all three molars crown can be scored as small. Measurements of vestibularlingual dimensions in automated digital mode are conducted for the second molar scans and are presented in Table 1 for images obtained by intraoral scanners Trios (3Shape) and CEREC Omnicam (Sirona).We are not presenting measurement accuracy assessments in the current article as we would like to have high resolution micro-CT scan as a reference for such comparisons. The only tooth from Shengavit subjected to micro-tomographic study (Skyscan 1174, Bruker) was a morphologically different tooth the first premolar. Thus further scanning is planned, though postponed. Manually obtained parameters can hardy serve as reference for several reasons: there are some methodological differences in manual and digital techniques, manual measurement accuracy is limited by one tenth of millimetre and manual measurements do not show enough for such comparisons consistency between several attempts to estimate the same parameter on the same tooth even when measured by one person, requiring calculations of inter- and intra-observer errors (Naidu and Freer, 2013).

\subsection{D Reconstruction}

Positioning of sample in CBCT scanning was found to have an effect on image shape. Thus a series of higher resolution CBCT scans obtained in a single sample position gave a better outcome.

\begin{tabular}{l|ccc}
\hline \hline \multicolumn{4}{c}{ Automated digital odontometry } \\
\hline Molars, Shengavit & M1 & M2 & M3 \\
\hline VL max, mm; (trios) & 9.86 & \\
section Nr. (out of 100) & 26 & \\
VL max, mm; (omni) & 9.85 & \\
section Nr. (out of 100) & 30 & \\
VL average, mm; (trios) & 7.665 & \\
VL average, mm; (omni) & 7.325 & \\
\hline \multicolumn{4}{c}{ Manual odontometry } \\
\hline MD max, mm & 10.5 & 10.6 \\
VL max, mm & 9.7 & 9.4 \\
\hline \multicolumn{4}{c}{ Attrition level; M. Gerasimov, 1955 } \\
\hline \multicolumn{4}{c}{34} \\
\hline \hline
\end{tabular}

Table 1. Absolute dimensions and attrition level of molars, Shengavit

The applied approach provided for obtaining a complete 3D image with higher resolution which as well as better results in reconstruction (Maret et al., 2014; Sang et al., 2016) (Figure 10 a). The obtained virtual reconstruction allowed prototyping on the sample for study and educational purposes (Figure 11). The studied sample volume and quality of the obtained images allowed performing virtual reconstruction of the complete mandible (Figure $10 \mathrm{~b})$.
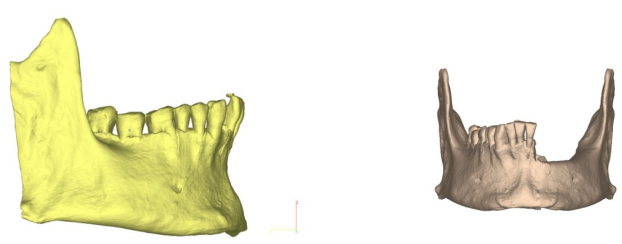

Figure 10. Final view of mandible fragment (a left) and reconstruction of the whole mandible (b right))

The results of incisor segmentation have resulted its printing out on a 3D printer serving for technological processes in dental practice (Figure $11 \mathrm{~b}$ ), thus the tooth can be substituted (restored) on the finding and can be used for further studies. On this example we can see how 3D imaging and processing techniques can serve for preservation of information regarding unique anthropological findings. Similar approaches can be suggested for educational purposes or museum funding.
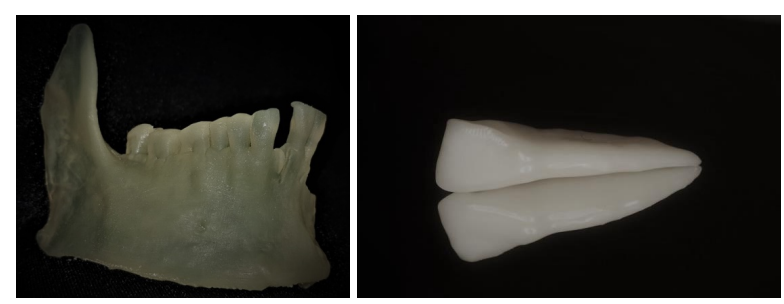

Figure 11. Final view of mandible fragment (a left) and reconstruction of the whole mandible (b right))

Figure $11 \mathrm{a}, \mathrm{b}$. Printed mandible right part (a left) and lower lateral incisor (b right). 


\section{CONCLUSION}

3D imaging techniques provide obtaining and storage of information, which has a potential to serve for substitution and restoration of materials referring to archaeological and palaeoantropological studies, serving as well as museum piece or training material. Image processing techniques serve for data extraction, resolution and format adjustments, image combining and prototyping, providing restoration of objects for further odontological descriptions or odontometry.

From our point of view similar procedures have to become a routine part of anthropological research in order improve methods of studies and preserve data referring the studied material for future generations of explorers.

\section{ACKNOWLEDGEMENTS}

The reported study was funded by Russian Foundation for Basic Research (RFBR) according to the research project 17-29-04509.

\section{REFERENCES}

Ahangar et al., 2019 Ahangar, P.; Cooke, M.E.; Weber, M.H.; Rosenzweig, D.H. Current Biomedical Applications of 3D Printing and Additive Manufacturing.Appl. Sci.2019,9, 1713.

Benazzi et al., 2011. Benazzi S., Fiorenza L., Kozakowski S., Kullmer O. Comparing 3D Virtual Methods for Hemimandibular Body Reconstruction. Anatomical Record. Vol. 294, pages 111625, doi: 10.1002/ar.21410.

Brown et al., 2018 Brown, G., Currier, G., Kadioglu, O. and Kierl, J., 2018. Accuracy of 3-dimensional printed dental models reconstructed from digital intraoral impressions. American Journal of Orthodontics and Dentofacial Orthopedics 154, pp. 733739

Chibunichev et al., 2018. Chibunichev A., Knyaz V., Zhuravlev D., Kurkov, V. Photogrammetry for Archaeology: Collecting Pieces Together. ISPRS - International Archives of the Photogrammetry, Remote Sensing and Spatial Information Sciences. XLII-2, pages 235-240, doi: 10.5194/isprs-archives-XLII-2-2352018.

Fiorenza L., Yong R., Ranjitkar S., Hughes T., Quayle M. Mcmenamin P., Kaidonis J., Townsend G., Justin A., 2018. Technical note: The use of 3D printing in dental anthropology collections. American Journal of Physical Anthropology. Vol. 167, doi: 10.1002/ajpa.23640.

Forshaw, Roger, 2014. Dental indicators of ancient dietary patterns: Dental analysis in archaeology. British dental journal. Vol. 216, pages 529-35, doi: 10.1038/sj.bdj.2014.353. Irish, J. D. and Scott, G., 2016. A Companion to Dental Anthropology. Wiley Blackwell. ISBN 978-1-118-84543-1

A.V. Gaboutchian, V.A. Knyaz, N.A. Leybova. Measurements Of Cuspal Slope Inclination Angles In Palaeoanthropological Applications. The International Archives of the Photogrammetry, Remote Sensing and Spatial Information Sciences, Volume XLII2/W4, 2017 2nd International ISPRS Workshop on PSBB, 1517 May 2017, Moscow, Russia, doi:10.5194/isprs-archives-XLII-2W4-185-2017
Gaboutchian, A. V. and Knyaz, V. A.: 3D IMAGES FOR AUTOMATED DIGITAL ODONTOMETRY, Int. Arch. Photogramm. Remote Sens. Spatial Inf. Sci., XLII-2/W18, 5357, https://doi.org/10.5194/isprs-archives-XLII-2-W18-53-2019, 2019.

Knyaz, V.A., Zheltov, S.Yu.: Photogrammetric techniques for dentistry analysis, planning and visualisation. International Archives of the Photogrammetry, Remote Sensing and Spatial Information Sciences - ISPRS Archives Volume 37, 2008, Pages 783-788 2008 21st ISPRS International Congress for Photogrammetry and Remote Sensing; Beijing; China; 3 July 2008 - 11 July 2008;

Knyaz, V. A.: Image-based 3D reconstruction and analysis for orthodontia, Int. Arch. Photogramm. Remote Sens. Spatial Inf. Sci., XXXIX-B3, 585589, https://doi.org/10.5194/isprsarchivesXXXIX-B3-585-2012, 2012.

Le Luyer M, Coquerelle M, Rottier S, Bayle P., 2016. Internal Tooth Structure and Burial Practices: Insights into the Neolithic Necropolis of Gurgy (France, 5100-4000 cal. BC). PLOS ONE 11(7): e0159688. https://doi.org/10.1371/journal.pone.0159688

Mahajan 2019. Mahajan S. Role of Human Tooth Wear Analysis in Archaeology: A Review. Ancient Asia. Vol. 10, doi: 10.5334/aa.181.

Maret et al., 2014. Maret, D., Peters, O., Galibourg, A., Dumoncel, J., Esclassan, R., Kahn, J.- L., Sixou, M. and No, T., 2014. Comparison of the accuracy of 3-dimensional cone-beam computed tomography and microcomputed tomography reconstructions by using different voxel sizes. Journal of Endodontics.

Martin et al., 2017. Martin R., Hublin J-J., Gunz P., Skinner M. The morphology of the enameldentine junction in Neanderthal molars: Gross morphology, non-metric traits, and temporal trends. Journal of Human Evolution. Vol. 103, pages 20-44, doi: 10.1016/j.jhevol.2016.12.004.

Naidu and Freer, 2013. Naidu, D., Freer, T. The evidence supporting methods of tooth width measurement: Part II. Digital models and intra-oral scanners. Australian orthodontic journal. 29. 164-9

Sam et al., 2019. Sam A., Currie K., Oh H., Flores-Mir C., Lagravere-Vich M. Reliability of different three-dimensional cephalometric landmarks in cone-beam computed tomography: A systematic review. The Angle Orthodontist. Vol. 89, doi: 10.2319/042018-302.1.

Sang et al., 2016 Sang, Y.-H., Hu, H.-C., Lu, S.-H., Wu, Y., Li, W.-R. and Tang, Z.-H., 2016. Accuracy assessment of threedimensional surface reconstructions of in vivo teeth from conebeam computed tomography. Chinese Medical Journal Vol. 129, pp. 1464.

Scianna and La Guardia, 2018. Scianna, A., La Guardia M. 3D Virtual CH Interactive Information Systems for a smart web browsing experience for desktop PCs and mobile devices. The International Archives of the Photogrammetry, Remote Sensing and Spatial Information Sciences, Volume XLII-2, 1053-59, https://doi.org/10.5194/isprs-archives-XLII-2-1053-2018

Simonyan H., 2015. The Archaeological Site of Shengavit: An Ancient Town in the Armenian Highland. Fundamental Armenology (Yerevan), No.1, pp. 134-168.

Simonyan H., 2018. Shengavit. Yerevans Ancient Settlement. Yerevan 5, Collection of Scientific Articles, pp. 3-13.

Skinner et al., 2008. Skinner M., Wood B., Boesch Ch., Olejniczak A., Rosas A., Smith T. Hublin J-J. Dental trait expression 
at the enamel-dentine junction of lower molars in extant and fossil hominoids. Journal of human evolution. Vol. 54, pp 173-86, doi: 10.1016/j.jhevol.2007.09.012.

Uldin, 2017. Uldin, T., 2017. Virtual anthropology a brief review of the literature and history of computed tomography. Forensic Sciences Research 2(4), pp. 165173

Vasilyev et al., 2019. Vasilyev S.V., Vasilyeva O.A., Galeev R.M., Dyuzheva O.P., Novikov M., Chichaev I.A., Yatsishina E.B. 3D reconstruction of the ancient Egyptian mummy skeleton from the Pushkin state museum of fine arts (i,1 1240) The International Archives of the Photogrammetry, Remote Sensing and Spatial Information Sciences, Volume XLII-2/W12, 2019, P. 225-232,https://doi.org/10.5194/isprs-archives-XLII-2W12-225-2019.

Vnuk et al., 2019. Vnuk V., Ippolitov E.V., Kamaev S.V., Kozlov S.V., Markov M.A., Novikov M.M., CherebyloS.A. Modernization of the Laser Stereolithography Sets LS-series. Photonics, Vol. 13 (6) 2019, 538-543, doi: 10.22184/19937296.FRos.2019.13.6.538.543

Zubov, 1968. Zubov A.A., Odontologiya. Metodika antropologicheskikh issledovaniy, Moscow, Nauka, 200 p.

Zubov, 2006. Zubov A., 2006. Methodological Handbook for Anthropological Analysis of Odontological Materials. ETNOONLINE, Moscow 